\title{
La Transformation moderne des Courants alternatifs en Courants continus. Les Redresseurs à Vapeur de Mercure.
}

\author{
Par V. SYLVESTRE, Ingénieut A. M. el I. E. G.
}

\begin{abstract}
Les redresseurs à vapeur de mercure représentent la solution la plus élégante qui ait été trouvéc du problème de la transformation des courants alternatifs en courant continu. Les inventeurs sont partis des propriétés bien connues des lampes à vapetur de mercure Cooper-Hewitt, qui alimentées par des courants alternatifs ne laissent passer le courant que dans un seul sens, anode-cathode, el sont arrivés da consiruire des appareils capables de débiter du courant continu d'intensités variant de 200 à 1000 ampères, à des tensions dépassant 1.500 volts. Ces redresseurs sont employes couramment aujourd'hui dans la pratique industrielle:

Monsieur Maurice Leblanc disait en 1913, au Congrès des Ingénieurs Electriciens d’Angleterre et de France tenu d̀ Paris :

"Il y a quelques années, en prenant possession de la présidence à laquelle avait bien voulu m'appeler " la Société Internationale des Electriciens, je disais aux jeunes ingénieurs qui m'entouraient:

"Croyez-moi, il n'y a plus rien à faire avec l'indnction, c'cst dans les tubes à vide qu'il faut chercher.

"Les progrès rapides de la soupape à mercure Cooper-Hewill permettent de prévoir que, dans un " avenir peu éloigné, il sera facile de redresser, avec elle, les courants des plus grands allernateurs et de les " transformer en courants continus à haute tension."

Depuis cette époque, la Société Anonyme Brown Boveri et The Westinghouse Cooper Hewill Co (2) sont parvenues à solutionner industriellement cette question (1).

Le but du présent article est de donner aux lecteurs de la Houille Blanche, des renseignements sur la théorie, le fonctionnement et les diverses applications de ces nouvelles machines, actuellement parfaitement au point el dont un certain nombre assure depuis plus de six années des services très sévères, tels que ceux: des laminoirs et des sous-stations d'alimentation de chemins de fer et tramways.
\end{abstract}

La question soulevée déjà en 1880 concernant l'application des courants continus et des courants alternatifs n'est pas encore résolue aujourd'hui. Selon toutes prévisions, elle n'aboutira jamais à une solution définitive, car les deux systèmes de courant présentent des avantages militant en leur faveur pour une application ou l'autre. Pour le transport à grande distance de l'énergie électrique par exemple, le courant alternatif par suite de sa facilité de transformation a supplanté incontestablement le courant continu. D'un autre côté cependant, le courant continu convient peut-être mieux que le courant alternatif monophasé à la commande des tramways ou des chemins de fer ; il est employé principalement aussi, sous une forme appropriée, pour les réactions électrolytiques. Il existe enfin une série d'appareils, comme par exemple les lampes à ampoules de quartz, les commandes d'engins spéciaux, le triage magnétique des métaux, etc... pour lesquels le courant continu est utilisé de préférence.

Par sujte de la grande extension prise par le courant alternatif, il a été nécessaire de bonne heure de le transformer en courant continu ; jusqu'à présent on réalisait cette transformation à l'aide de machines rotatives moteur-générateur, convertisseur en cascade ou commutatrice.

Depuis longtemps, le désir a été émis en électrotechnique d'obtenir avec le moins de pertes possible les transformations d'énergie.

L'appareil réalisant d'une façon parfaite cette condition est le transformateur pour courants alternatifs, avec lequel on obtient la transformation de courants alternatifs à haute tension en courants alternatifs à basse tension ou inversement avec un rendemẹnt élevé, atteint par aucune autre machine électrique. Les inconvénients des convertisseurs rotatifs de courants alternatifs en courants continus employés jusqu'ici, tels que les pertes par frottement, le bruit et l'exigence d'une surveillance spéciale, rendaient particulièrement désirable la création d'un convertisseur n'utilisant pas de travail mécanique.

Ce convertisseur idéal est réalisé aujourd'hui déjà en grande partie par le redresseur à vapeur de mercure. L'exposé qui va suivre tend à donner une description détaillée de toutes les particularités pratiques des redresseurs à vapeur de mercure à petit et grand débit qui, malgré le grand nombre de leurs applications, sont encore peu connus en électrotechnique.

\section{DONNÉES THÉORIQUES (3)}

L'arc électrique. - Si l'on rompt un circuit électrique, il se produit toujours à l'endroit de la rupture un arc lumineux, qui, par suite de sa luminosité et de la quantité de chaleur qu'il dégage, a été de tous temps d'un grand

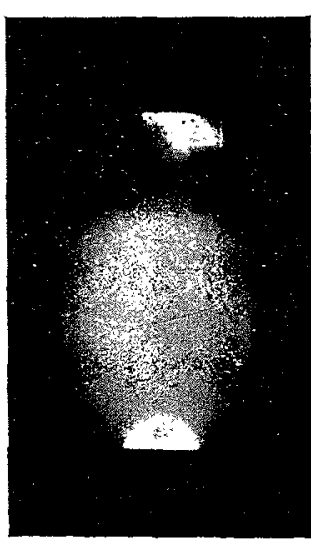
intérêt. A part une série de qualités remarquables, l'arc électrique possède encore la propriété de ne laisser passer le courant que dans un sens, c'est-àdire qu'avec du courant alternatif, il ne peut être traversé que par les impulsions de courant de même sens. Cet effet de soupape électrique, analogue à l'effet d'une soupape de pompe, est spécialement réalisé par un arc lumineux au mercure jaillissant dans un espace vide et constitue le principe fondamental du redresseur à décrire.

Afin de mieux comprendre les phénomènes qui se passent, il est bon d'exposer et d'expliquer sommairement le phénomène de l'arc électrique.

Le moyen le plus simplé de produire un arc est l'allumage par contact dans lequel les électrodes sous tension sont approchées jusqu'à se toucher puis à nouveau séparées. Un arc formé dans l'air atmosphérique par du courant continu, d'une longueur attejgnant généralement 1 à 2 centimètres, porte le pôle positif, anode, et le pôle négatif, cathode, au blanc incandescent, les deux pôles étant reliés par l'arc électrique lui-même. Suivant sa longueur, il est nécessaire pour maintenir convcnablement l'arc, de 30 à 40 volts et d'au moins 3 ampères. Dans le vide, il est évident qu'à tensions égales, les électrodes peuvent être plus écartées que dans l'air, elles présenteront en outre dans leur façon de se comporter des différences essentielles. L'arc forme une auréole à l'anode et en élève uniformément la température sans cependant

(1) Voir Revue B. B. C., 1919, No 5 à 9.

(2) Actuellement Hewittic Elecíric $\mathrm{C}^{\circ}$, à Suresnes (Seine).

(3) Voir Revue B. B. C., 1919, Nos 5, 6, 7, 8 et 9 . Dr. Sch; (De) 
que celle-ci solt assez forte pour produire une évaporation ; à la cathode par contre, on remarque une tache claire, blanche incandescente, appelée tache de cathode (base de l'arc), qui se déplace avec une grande rapidité à sa surface en décrivant des courbes complètement irrégulières. Avec dès électrodes liquides, par exemple de mercure, il se forme sous la tache de cathode un profond cratère, duquel surgit un flot immense de vapeur de métal. Dans le cas d'un vide élevé on aperçoit au-dessus du cratère une flamme assez haute appelée flamme négative (fig. 1) facile à influencer par le champ magnétique et qui, comme nous le montrerons, peut jouer un rôle défavorable en particulier dans les redresseurs à vapeur de mercure. La colonne lumineuse positive est remarquable par le fait qu'elle sort directement de l'anode et. reste compacte jusqu'à ce qu'elle atteigne la cathode où elle laisse libre un espace sombre. Dans les lampes à vapeur de mercure, la source lumineuse est formée par cette colonne lumineuse positive.

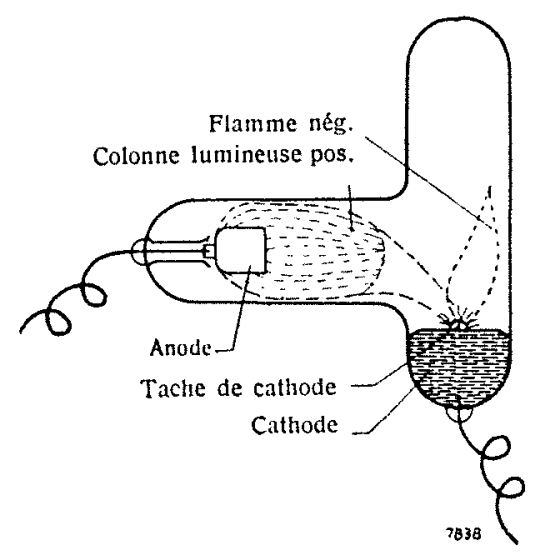

Fig. 1.

Pour expliquer ce phénomène, on se base sur la théorie admise après de nombreuses observations, disant que Ie transport de l'électricité dans les gaz se fait par des charges élémentaires positives et négatives, appeléesions. La charge des ions positifs est toujours liée à l'atome chimique, tandis que la charge élémentaire négative, l'électron, n'apparaît pas comme une masse pondérable ; la molécule neutre admet par contre indifféremment des charges positives ou négatives.Dans l'atmosphère de l'arc électrique de mercure se trouvent aussi bien des particules dissociées que des particules neutres.

En un point de la surface de la cathode chauffé jusqu'au blanc incandescent sous l'influence du champ électrique, des électrons libres s'échappent vers l'anode avec une vitesse croissante. Pendant son parcours l'électron doué d'une énergie cinétique suffisante peut, par sa collision avec des molécules neutres, séparer les charges électriques positives et négatives (ionisation par choc). Il se crée ainsi des ions positifs et des électrons négatifs nouveaux qui élèvent la conductance de l'espace de vapeur.

Les ions positifs existants et ceux nouvellement formés se précipitent vers la cathode où, par le bombardement intense d'un très petit espace, ils échauffent la surface jusqu'au blanc incandescent et contribuent ainsi de leur côté au maintien de la décharge.

Il est donc important de remarquer que la formation d'un arc électrique dépend essentiellement de l'existence d'électrons libres, comme c'est le cas par exemple lorsque la cathode est chauffee jusqu'au blanc incandescent sous l'influcnce d'un champ électrique. Cette constatation fondamentale peut être mise en évidence par les expériences suivantes :

La figure 2 représente un récipient en verre dans lequel on a fait le vide et contenant 2 électrodes principales $(a)$ ct $(b)$ et une électrode auxiliaire (c). Entre l'électrode solide $(a)$ en fer ou en graphite et l'électrode liquide de mercure $(b)$. un arc électrique doit être allumé. L'électrode $(c)$ prévue dans ce but peut, par un léger basculement de tout le récipient, entrer momentanément en contact avec l'électrode (b), d'où formation d'un arc avec cette dernière comme cathode. La condition exigeant qu'en premier lieu un point de la surface de (b) soit chauffé jusqu'au blanc incandescent est donc satisfaite. Par rapport au circuit I l'électrode (b) peut, suivant la connexion de la source de courant continu, être une anode ou une cathode. Il est cependant évident que, si $a$ est négatif et $b$ positif, il ne se formera aucun arc entre eux sous l'effet de la tension seule, même portée à plusieurs milliers de volts. Au contraire, 20 à 30 volts suffisent si $(a)$ et $(b)$ sont connectés au circuit, $(a)$ comme anode et $(b)$ comme cathode.

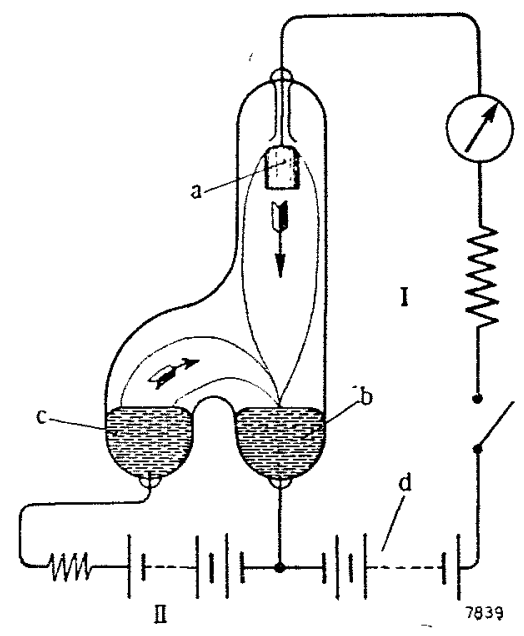

Fig. 2.

Les électrons peuvent seulement dans ce dernier cas ioniser l'espace de vapeur $(a-b)$. Il faut encore remarquer que même, si les électrodes principales sont connectées correctement,, $(b)$ négatif et $(a)$ positif, l'arc ne se produira pas si par rapport au circuit d'allumage II, (b) est positif et (c) négatif.

Il en résulte que la méthode par allumage indirect, la seule appliquée dans les redresseurs à vapeur de mercure, ne peut l'être utilement que si l'électrode principale de mercure est une cathode aussi bien dans le circuit d'allumage que dans le circuit principal, c'est-à-dire dans le cas seulement où elle est connectée au pôle négatif des deux circuits.

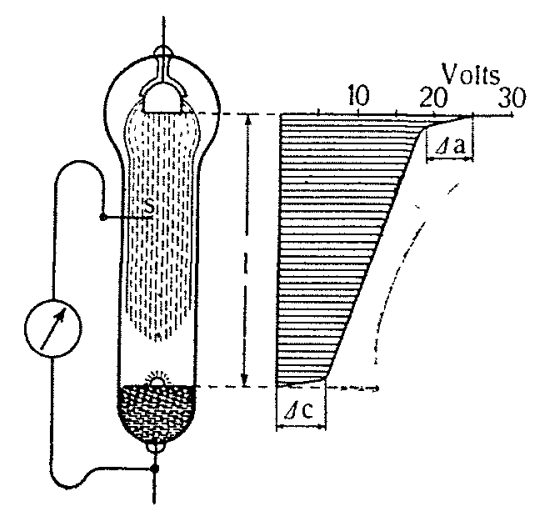

Fig. 3.

Chute de tension dans l'arc électrique. - La chute de tension mesurée dans un arc se compose de trois parties : la chute de tension à l'anode $-a$, celle de la cathode $-c$, et la tension absorbée par l'arc proprement dit (figure 3 ).

Pour mesurer ces différentes valeurs on peut introduire dans 
l'arc, aux endroits où se produit un changement de tension, une sonde en platine. Less sauts brusque., do la courbe représentent les chutes de tension à l'anode et à la cathóde, et la partiè du milieu qui tombe lentement, la chute de tension dans l'arc. La ehute de tension aux électrodes est assez constante et pratiquement indépendante du matériel, de l'intensité du courant ainsi que de la pression du gaz: Pour une anode en fer $\Delta a=6,5$ volts, et pour une cathode de mercure $\Delta . c=5,5$ volts, soit au total 12 volts. Par contre; la différence de potentiel est très variable, par centimètre de longueur de l'arc, suivant la pression du gaz, l'intensité du courant et la section libre de l'arc. On peut dire que cette chute de tension par unité de longueur de lá veine lumineuse $=$ volt/cm, est d'autant plus faible que la section de l'arc et l'intensité du courant sont plus fortes et que la pression sous laquelle l'arc brûle est plus faible.

Dans des tubes d'éclairage longs et étroits aveć une pression de 1 à $2 \mathrm{~m} / \mathrm{m}$ de colonne de mercure et une consommation de courant de seulement 3 à 4 ampères on mesurera 0,8 à 1,2 volt de chute de tension par centimètre de longueur de l'arc. Dans les redresseurs ayant une pression beaucoup plus basse, une plus grande section et recevant un courant de plusieurs centaines d'ampères, la valeur définie plus haut s'abaisse à 0,1 volt et au-dessous.

En appliquant les valeurs obtenues ci-dessus à un redresseur avant un are lumineux de $60 \mathrm{c} / \mathrm{m}$ de longueur, la chute totale sera de :

$\varepsilon=\lrcorner a+\lrcorner c+1$ volt $/ \mathrm{cm}=6,5+5,5+60 \times 0,1=18$ volts

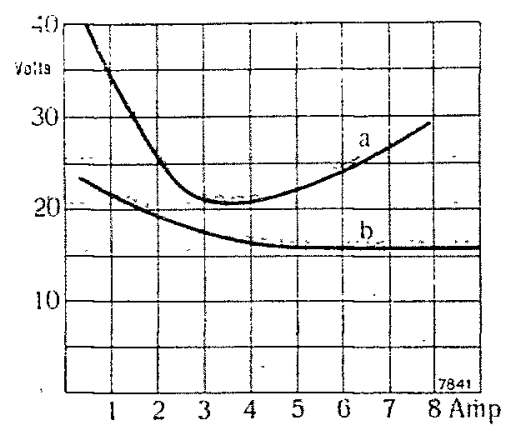

Fig. 4.

Cette tension minimum est celle dépensée pour allumer l'arc. En réalité, la tension d'allumage sera un peu plus haute, car elle doit vaincre la résistance initiale plus élevée du parcours de l'arc. La relation entre le courant et la tension est représentée sur la figure 4. La courbe supérieure (a) à été obtenue avec une lampe à vapeur de mercure, la courbe inférieure $(b)$ avec un redresseur. On remarque qu'avec les lampes, à un accroissement de l'intensité correspond premièrement un abaissèment de la tension d'électrodes qui, aprês le passāge d'un minimum bien marqué, croît de nouveau.

Dans la première partie de la courbè, la conductance du chemin parcouru par le courant augmente par suite de l'accroissement du dégagement de la vapeur. Dans la seconde partie, par contre, l'élévation de la pression de la vapeur cause aussi une augmentation de la chute de tension par centimètre de longueur de la colonne lumineúse, et produit une augmentation de la différence de potentiel aux bornes de l'ärc. Avee le redresseur, la pärtí gauche seulement de la caractéristique vólti-ampères peut être normalement examinée, l'autre partie étant tellemerit aplatie que l'on peut parlex pratíquement d'une constante de chute de tension dans l'arc limineux pour toutos les valeurs du courant. Pour des valeurs croissantes de l'inteńsité du courant, In caractéristique de clute de teinsion sera désignêe aussi par caractéristique négative. Les circuits électriques prêsentant unte chute de tension semblable ne peuvent être coninectés en parallèle sans dispositif spècial, car un des circuits offrant accidentellenthent une chute de tension légèrement plus faible, appelleta la totalité du courant. Afin de pouvoir cependent brancher en parallèle des arcs électriques de redresseurs, chose exigée pour l'augmentation de la puissance, jl est nêceessaire d'insérer dans leurs circúts dẹs résistances ohmiques ou inductives, à l'aide desquelles la catat. téristique négative deviendra une carnctéristique posllive et la tension des circuits branchés en parallète croîtra quand les courants augmenteront. Il suffil déjà, comme nous le verrons, d'intercaler de petites bobines de self pour réaliser aisément un couplage en parallèle de rèdresseurs.

La connaissance de la constante pratique de chute de tension est nécessaire pour l'appréciation de la perte dans un redresscur. La mesure peut avoir lieu avec du courant continu ou du courant alternatif. Dans le premier cas, on branche, en parallèle avec les électrodes, un voltmètre dont la lecture directe donne la chute totale $\zeta$ quj, multipliée par le courant traversant mesuré simultanément $I_{c}$ donne la perte à l'intérieur du redresseur. Si l'on emplcie du courant alternatif pour celte mesure, on procédera en sens contraire, car un wat tmètre donnera la perte de travail $\mathrm{L}_{\mathrm{c}}$ et un ampèremètre le courant continu employé $i_{\mathrm{c}}$. Le quotient des deux donnera la chute de tension effective totale dans l'are lumineux (figure 5).

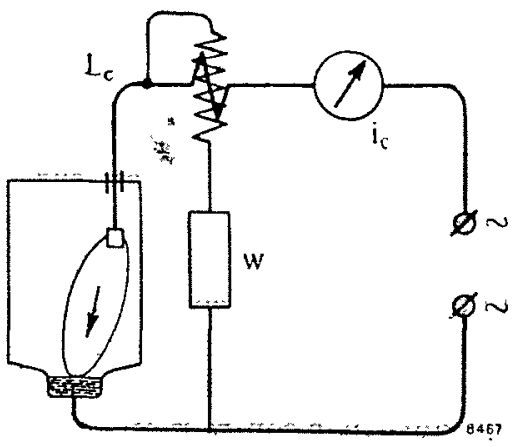

Fig. 5.

Cette méthode de mesure ne donne des valeurs exactes que pour des tensions de courant alternatif allant jusqu'à 250 volts. Pour des tensions plus élevées la résistance du wattmètre, intercalé dans le circuit devient trop grande et par conséquent la déviation correspondante très petite.

Effets de valve، - Nous avons vu que des électrons ne se déghagent d'une éleêtrode sous tension que si elle est chauffée au blatte incandescent. Par suite de lcur impulsion, ils contribuent à ioniser l'espace entre les électrodes et préparent le passage du courant sous fórme d'arre lumineux. Les électrons ne peuvent se forme" sur une électrode froide; si l'on place donc vis-à-vis l'une de l'aut* tre deux électrodes semblables, le passage du courant ne pournis avoir lieu que si l'électrode fortement chauffée est une cathode (car celle-ci seule dégage des électrons négatifs.); si l'on change la polarité, le passage du courant cessera. Si dans le circuit pritcipal I, du schéma de la figure 2 , on remplace la source de courant continu par une source de courant alternatiil de tension assez élevée, en maintenant dans le circuit auxiliaire II l'arc lumineux de courant continu ehauffant l'etelfor 
trode de mercure $(b)$, un arc luminedx sallumera de l'electrode $(a)$ a l'électrode $(b)$, chaque fois que $(a)$ scra positif et $(b)$ négatif. Entre temps, tout passage de courant cessera entre $(a)$ el (b). La figure 7 représente la forme du courant pour ủie tension sinusoïdale correspondante. Il ne faut pas s'imaginer que l'énergie correspondant à ces absences périodiques de courant soit anéantic, an contraire, son arrivéc dans le circuit correspondant et pour la période correspondante est suspendue. Des oscillogrammes montrent que pratiquement il ne passe pas de courant dans celte zone (a). On peut démontrer cependant, par des méthodes de mesures spéciales, que dans l'utilisation de l'arc lumineux au mercure comme valve clectrique (ou mieux valve d'électrons) il passe aussi un courant de retour qui croit avec la tension et l'intensité du courant emplové, ainsi qu'avec la température de lanode, sans cependant dépasser normalement quelques millianpères et par conséquent sanis porter préjudice au rendement de l'installation.

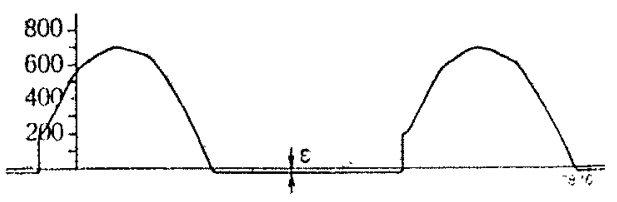

Fig. 6.

Unc anode en fer ou en graphile peut ètre portée jusqu'au rouge n̈tandescenit sans modifier pour cela la qualité de l'eff at de valve, Il est important que lo vide du récipient reste aussi élevé que possible, car' c'est la condition nécessaire pour que l'arc lumineux forme à l'anode une auréole régulière, sans élévation de temperature exagérée ou sans vaporisation locale de sa surface. Au contraire, avec une pression du gaz croissante et notamment dans les redresseurs encore peu cmployés, l'are lumineux a facilement la tendance de se concentrer en un point de la surface de l'anode, co yui peut causer par la vaporisation du métal et la formation d'une tache de cathode, un court-circuit. Gelui-ci consiste en une cessation rapide de l'effet de valve avec passage de courant dans les deux sens.

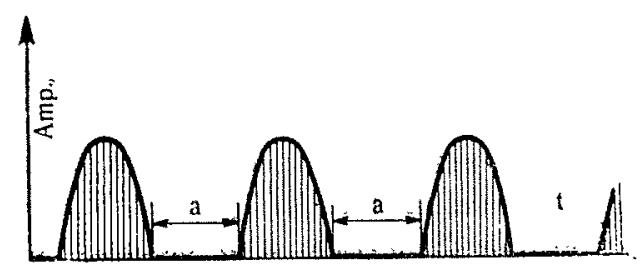

Fig. 7.

Ces court-circuits, si nuisibles dans le cas des redresseurs peuvent en plus de cclle exposée tout à l'heure, avoir encore d'autres origines. Des gouttelettes de mercure ruisselant sur l'anode peuvent en provoquer. Les rayons ultra-violets produisent le même effet et sont particulièrement abondant, dans l'arc lumineux au mercure où ils ont une action ionisante.

L'entrée en contact de l'anode avec la flamme négative déjà mentionnée, des surtensions subites, de forts accroissements de la pression du gaz en sont aussi les causes. Dans les redresseurs modernes, toutes ces influences sont éliminées par une construction approprice et susceptible encore de perfectionnements.
REDRESSEUR. - Type Rectigon de la Westinghouse Electric Co et Tungar de la General Eleciric Có.

Nous venons de voir dans le chapıtre précédent que lès électtrons se dégagent d'une électrode sous tension chauffée au blanc incandescent, et que l'effet de valve se produit si l'électrode portée au blanc est une cathode.

Les pelits redresseurs, construits en Amérique par la Westinghouse Electric Co, le Rectigon, et par la General Electric Co, le Tungar, ce dernier vendu en France par la Cie Thomson-Houston, sont basés sur ce principe.

Ces petits redresseurs sont employés à la charge des petites batteries d'accumulateurs ; ils sont généralement construits pour 12 ampères, 75 volts côté continu ; on en construit jusqu'à 100 ampères.

Principe. - Ce redresseur consiste essentiellement en un aulotransformateur et une ampoule montés dans une même caisse (schéma no 8). L'ampoule (fig. 9) qui, à première vue, ressemble à

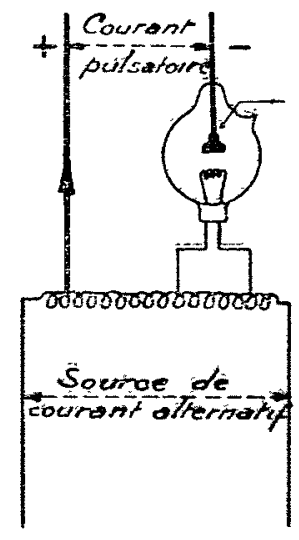

Fig. 8.

une lampe à incandescence, còntient un filament à bas voltage en tungstène, c'est la cathode, une électrode en graphite formant l'anode, placćes dans une atmosphère d'argon. Sa constitution est

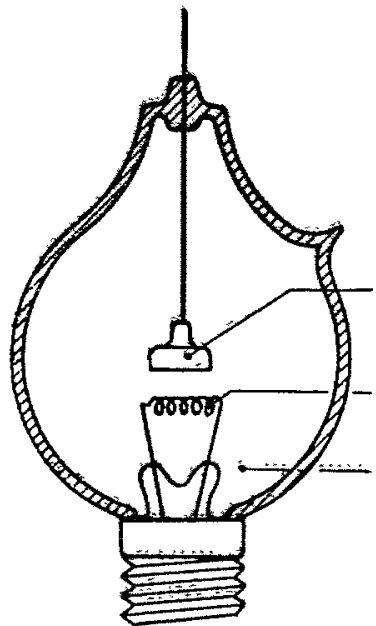

Fig. 9.

done analogue à celle de la valve de Fleming mais, grâce à la présence du gaz "Argon ", le mode de fonctionnement est complètement modifiè et l'intensité du courant redressé peut atteindre une bien plus grande valeur. La désignation "Tungar " de la General Electric $C^{\circ}$ est l'abréviation TUNGstc̀ne-ARgon. 
L'ampoule a la forme d'une lampe, la cathode est montée en dérivation (figure 8) sur une partie de l'enroulement de l'autotransformateur, ce dernier permettant d'adaptel la tension du secteur à celle désirée pour le courant redressé. distincts et non à l'aide d'un auto-transformateur ; de celte façon le circuit d'ulilisation est complètement isolé du secteur. Il cn résulte que l'on peut charger sans inconvénient des balteries mal isolées ou ayant un pôlc à la terre et que l'on peut toucher par

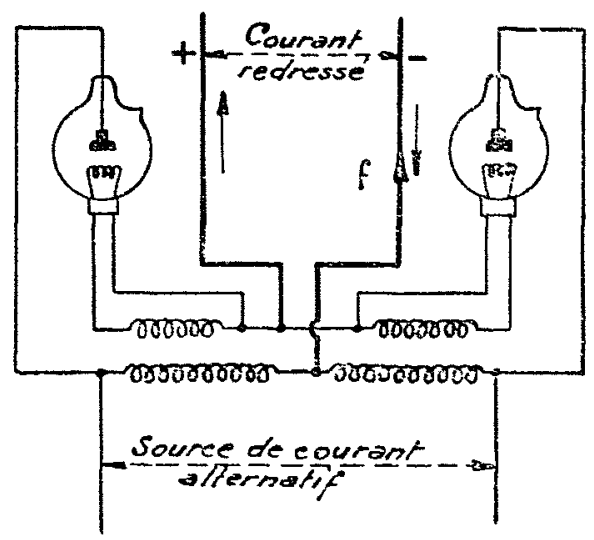

Fig. 10 .

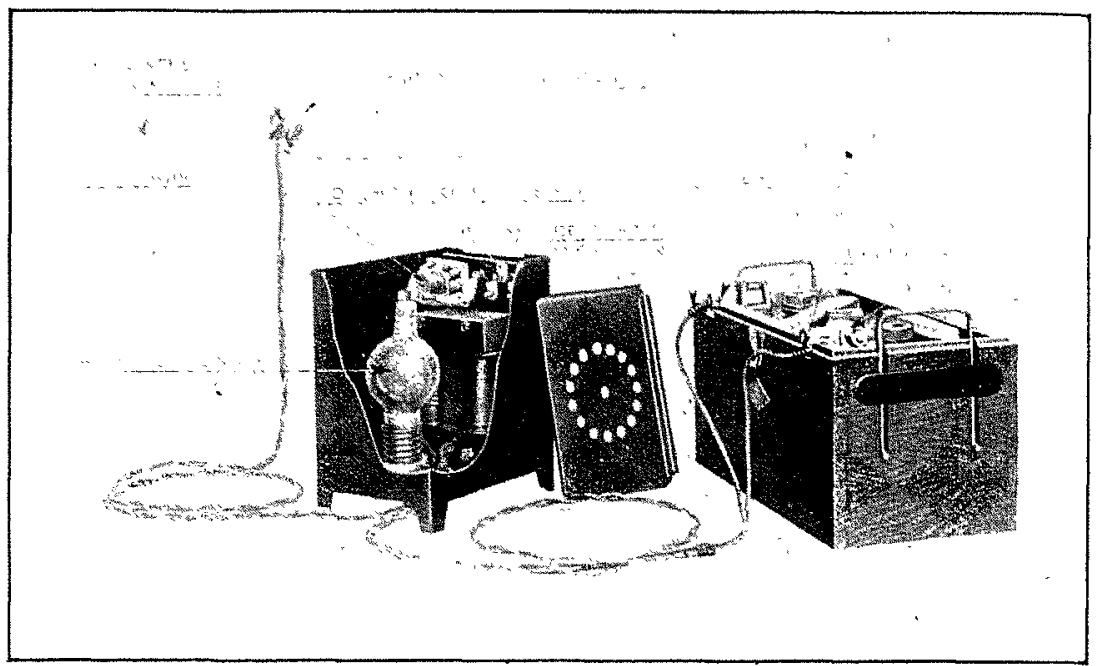

Fig. 12. - Redresseur "Tungar " chargeant une petite batleric d'accumulateurs.

inadvertance au circuit d'utilisation sans ressentir de secousses. La durée des ampoules est aussi longue que celle des lampes à incandescence ordinaire. Généralement une ampoule peut assurer pendant 600 à 800 heures un service chargé, pendant 800 à 1.000 heures un service normal, pendant 1.000 à 1.600 heures en service peu chargé. On a vu des ampoules durer jusyu'à 2.000 heures. L'appareil peut supporter des surcharges importantes. Ce petit redresseur peut fonctionner sans surveillance; il peut charger pendant la nuit une batterie utilisée pendant le jour; il permet ainsi, dans bien des cas, de faire l'économie d'une batterie de rechange (figure 12).

Les types normaux de redresseurs Tungar sont los suivants :

a) Type de "Garage privé ".

Ce type est concu spécialement pour les besoins de l'automobilisme. Il permet de charger une palterie de démarrage à 3 élémenls au régime de 5 ampères, ou une ballerie de 6 ćléments au régime de 3 ampères.

\section{b) Type dit téléphonique.}

La figure $n^{\circ} 8$ indique le montage d'un redresseur Rectigon sur auto-transformateur utilisant seulement la moitié des ondes de la courbe de la force électromotrice. La figure no 10 donne un montage utilisant la totalité des ondes.

La Cie Thomson-Houston alimente toujours les redresseurs Tungar (fig 11) à l'aide d'un transformateur à deux enroulements
Ce type est plus spécialement établi pour la charge des batteries centrales de 12 éléments au plomb. Son régime moyen de charge est de 5 ampères. On peut utiliser des régimes plus petits en réglant la résistance d'un rhéoslat placé sur l'apparcil.

(A suivre.) 